\title{
Susceptibilidad a antibióticos de cepas paraguayas de Helicobacter pylori aisladas de pacientes con enfermedad gastro-duodenal
}

\author{
N orma Fariñala Elena Kasamatsu², Margarita Samudiola,b, \\ Miryam Morán²a, Rosa Sanabria1a, Florentina Laspina ${ }^{1 a}$. \\ Antimicrobial susceptibility of \\ $H$ pylori strains obtained \\ from Paraguayan patients
}

Background: Antimicrobial resistance is one of the main obstacles for an effective eradication of $\mathrm{H}$. pylori infection. Aim: To determine the susceptibility of $\mathrm{H}$. pylori strains obtained from gastric biopsies to metronidazole, clarithromycin and amoxicillin. Material and methods: Susceptibility to metronidazole, clarithromycin and amoxicillin was determined using E-test in 46 isolates of $\mathrm{H}$. pylori obtained from gastric biopsies of 54 adult patients. Results: Thirty three percent of isolates were resistant to metronidazole and $2 \%$ were resistant to clarithromycin and amoxicillin. One isolate was resistant to metronidazole and clarithromycin. Conclusions: The antimicrobial susceptibility of these strains of H. pylori obtained from Paraguayan patients, may help to decide the initial therapy to eradicate this infection (Rev Méd Chile 2007; 135: 1009-14).

(Key w ords: Anti-bacterial agents; Biopsy; Helicobacter pylori)

\author{
Recibido el 13 de septiembre, 2006. Aceptado el 27 de febrero, 2007. \\ ${ }^{1}$ Departamento de Microbiología, ${ }^{2}$ Departamento de Patología, Instituto de Investigaciones \\ en Ciencias de la Salud. Universidad Nacional de Asunción, Paraguay. \\ aBioquímico \\ bDoctor en Parasitología
}

$\mathrm{H}$ elicobacter pylori es un patógeno del tracto gastro-duodenal humano que está asociado a gastritis crónica, úlcera péptica, linfoma de MALT (mucosa associated lymphoid tissue) y cáncer gástrico $^{1}$. Fue aislado por primera vez de la mucosa gástrica del ser humano en 1982, por los investigadores australianos Marshall y Warren ${ }^{2}$.

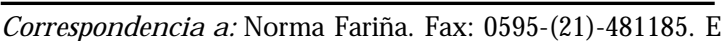
mail: microbiologia@iics.una.py
H. pylori es un bacilo Gram negativo, móvil, curvo espiralado, microaerofílico y se caracteriza por la producción de ureasa, una enzima muy activa característica de este microorganismo, que le permite a la bacteria colonizar y permanecer en la capa mucosa que cubre la cavidad gástrica. $\mathrm{H}$. pylori se encuentra ampliamente distribuido a nivel mundial y su incidencia es aún mayor en países en vías de desarrollo, incluyendo Paraguay $^{3,4}$. Desde 1994, la IARC (Agencia Internacional para la Investigación del Cáncer de la 
Organización Mundial de la Salud) lo ha incluido entre los agentes carcinógenos tipo 1, constituyéndose en una de las especies bacterianas de mayor interés en patología humana ${ }^{5}$.

Actualmente, existe consenso internacional para el tratamiento de todo paciente infectado con $\mathrm{H}$. pylori como una medida de prevención del cáncer gástrico, ya que la infección por esta bacteria induce cambios inflamatorios crónicos progresivos de la mucosa gástrica, con evolución a gastritis atrófica multifocal, metaplasia intestinal, displasia y cáncer gástrico ${ }^{6,7}$. Las pautas de tratamiento para la erradicación de $\mathrm{H}$. pylori combinan 2 ó 3 antimicrobianos (amoxicilina, claritromicina, metronidazol, tetraciclina) y una droga que disminuya la secreción clorhídrica del estómago. Las monoterapias no han demostrado utilidad clínica y se recomienda el empleo de terapias dobles, triples o incluso cuádruples. La triple terapia más comúnmente utilizada incluye un inhibidor de la bomba de protones, omeprazol, y dos antibióticos, claritromicina y metronidazol o amoxicilina $a^{3,8,9}$.

Las cepas de H. pylori son susceptibles in vitro a un número elevado de antibióticos, aunque muchos de ellos no son útiles in vivo. Esto se debe a que el antibiótico no se distribuye adecuadamente en los sitios profundos de la mucosa gástrica, donde esta bacteria coloniza, o porque el antibiótico se inactiva a $\mathrm{pH}$ ácido, condición presente en el estómago, o porque se seleccionan mutantes resistentes durante el tratamiento ${ }^{10}$. La aparición de resistencia a metronidazol ha emergido en el mundo entero, poniendo en peligro el éxito terapéutico ${ }^{3,11-14}$. La resistencia a claritromicina es variable ${ }^{11}$ y a amoxicilina es significativamente menor, registrándose sólo casos aislados de resistencia ${ }^{3}$.

Para la determinación de la sensibilidad de $\mathrm{H}$. pylori a los antimicrobianos, el método de dilución del antibiótico en agar es el método de referencia según el National Committee for Clinical Laboratory Standards (NCCLS) ${ }^{15}$. El E test (método epsilómetro) tiene muy buena correlación con el método de referencia ${ }^{16}$ y su uso está recomendado por la British Society for Antimicrobial Chemotherapy (BSAC) ${ }^{9}$.

El conocimiento de la susceptibilidad antibiótica de $\mathrm{H}$. pylori a nivel local, tiene importancia para la selección de un apropiado régimen de tratamiento. En Paraguay no se realiza cultivos de H. pylori para diagnóstico, ni existen estudios publicados sobre resistencia a antibióticos, por lo que nuestro objetivo fue conocer el patrón de sensibilidad de cepas de $\mathrm{H}$. pylori aisladas de biopsias gástricas a los antibióticos más utilizados en la actualidad, metronidazol, claritromicina y amoxicilina.

\section{Material y MÉTOdo}

Pacientes. Mediante endoscopia digestiva alta se tomaron 6 biopsias gástricas, 3 de antro y 3 de cuerpo, a 333 pacientes adultos (rango: 18-88 años), de ambos sexos (56,5\% varones) con síntomas de enfermedad gastroduodenal que concurrieron al Departamento de Endoscopia Digestiva del Hospital de Clínicas de la Facultad de Ciencias Médicas de la Universidad Nacional de Asunción y del Instituto de Previsión Social, entre los años 2002 y 2003. A los pacientes se les tomó las muestras, previo consentimiento informado. Fueron excluidos aquellos pacientes que en las dos semanas anteriores habían recibido tratamiento antibiótico, compuestos de bismuto, inhibidores de la bomba de protones o antiinflamatorios no esteroides. Las muestras fueron congeladas a $80^{\circ} \mathrm{C}$ en $0,5 \mathrm{ml}$ de caldo Brucella suplementado con glicerol al $30 \%$.

Aislamiento e identificación. En 208 pacientes, de los 333 enrolados en este estudio, se confirmó la presencia de $\mathrm{H}$. pylori por estudio histológico. De éstos, se seleccionaron las biopsias de 60 pacientes, con diferentes tipos de lesión y grados de infección II (moderado) y III (severo). Para el cultivo fueron descongeladas 2 biopsias por paciente, una de antro y otra de cuerpo, las que fueron maceradas y sembradas en dos medios: Agar cerebro corazón suplementado con sangre de carnero al $10 \%$, adicionado de vancomicina, ácido nalidíxico y cicloheximida, y medio de agar cerebro corazón con el agregado de cloruro de 2,3,5 trifenil tetrazolio. Posteriormente fueron incubados en jarras de microaerofilia, utilizando sobres generadores de microaerofilia Genbox microaer (bioMérieux ${ }^{\circledR}$, Francia) a $35^{\circ} \mathrm{C}$ durante 10 días y observados cada 3 días. La identificación de la bacteria fue realizada por el pequeño tamaño 
de las colonias, la presencia de bacilos Gram negativos curvos, en S itálica o espiralados en la coloración de Gram, prueba positiva para ureasa y además oxidasa y catalasa ${ }^{9,17,18}$.

Determinación de la sensibilidad a los antibióticos. El inóculo fue preparado con cultivo de 2 ó 3 días en caldo cerebro corazón ajustado a una turbidez 3 a 4 en la escala de Mc Farland. La suspensión de bacterias resultante se hisopó en tres direcciones sobre placas de agar Müeller Hinton, suplementadas con sangre de carnero al 10\%, una vez secas, se colocó una tira de E test por cada placa de $10 \mathrm{~mm}$ de diámetro y se incubaron en atmósfera de microaerofilia durante 3 a 5 días.

La lectura de la concentración inhibitoria mínima se realizó en la intercepción de la tira con el vértice de la elipse. Como cepa control fue utilizada la cepa de H. pylori ATCC 43504 resistente a metronidazol (CIM $=64-256 \mathrm{mg} / \mathrm{l})$ y sensible a amoxicilina y claritromicina (CIM $=0,016-0,125$ $\mathrm{mg} / \mathrm{l})$. Los puntos de corte fueron los recomendados por la BSAC. Para el metronidazol CIM $\leq 4 \mathrm{mg} /$ l fue considerado sensible y $\mathrm{CIM} \geq 8 \mathrm{mg} / \mathrm{l}$ resistente. Para claritromicina y amoxicilina CIM $\leq 1 \mathrm{mg} / \mathrm{l}$ fue interpretado como sensible y $\mathrm{CIM} \geq 2 \mathrm{mg} / \mathrm{l}$ como resistente ${ }^{9}$.

\section{RESULTADOS}

El desarrollo de $H$. pylori se obtuvo en las muestras obtenidas de 54 pacientes y la determinación de la sensibilidad a los antibióticos fue posible en 46 aislamientos, por problemas de viabilidad o contaminación. El diagnóstico anatomopatológico de los 46 casos fue: 20 pacientes con gastritis no atrófica, 12 pacientes con úlcera duodenal, 12 pacientes con lesiones preneoplásicas y 2 pacientes con cáncer gástrico.

Los resultados de este estudio muestran que $32,6 \%$ ( $\mathrm{n}=15 / 46$ ) de los aislados de $\mathrm{H}$. pylori fueron resistentes a metronidazol y que 2,2\% (1/ 46) a claritromicina y amoxicilina.

En la distribución de frecuencias de aislamientos de acuerdo a la CIM, se puede observar que $43,5 \%$ de los 46 aislados presentó CIM a metronidazol $\leq 0,016$ $\mathrm{mg} / \mathrm{l}$ y que $28,2 \%$ presentó CIM $\geq 256 \mathrm{mg} / \mathrm{l}$ (Tabla 1 ). No se observaron cepas con valores de CIM cercanas al punto de corte. El aislado resistente a claritromicina presentó CIM de $16 \mathrm{mg} / \mathrm{l}$, de igual manera el aislado resistente a la amoxicilina presentó CIM de $16 \mathrm{mg} / \mathrm{l}$. Sólo un aislado de $\mathrm{H}$. pylori presentó resistencia simultánea a metronidazol y claritromicina.

Con relación a los tipos histológicos, la resistencia al metronidazol se observó en 30\% (6/20)

Tabla 1. Concentración inhibitoria mínima de aislados de H . pylori

\begin{tabular}{|lccc|}
\hline CIM $(\mathrm{mg} / \mathrm{l})$ & Metronidazol & Claritromicina & Amoxicilina \\
\hline 0,016 & $20(43,5 \%)$ & $43(93,4)$ & $45(97,8)$ \\
0,032 & $2(4,3 \%)$ & $1(2,2)$ & - \\
0,047 & $1(2,2 \%)$ & - & - \\
0,064 & $2(4,3 \%)$ & - & - \\
0,094 & $2(4,3 \%)$ & - & - \\
0,125 & $1(2,2 \%)$ & $1(2,2)$ & - \\
0,25 & $1(2,2 \%)$ & - & - \\
0,38 & $1(2,2 \%)$ & - & - \\
1 & $1(2,2 \%)$ & - & $1(2,2)$ \\
16 & - & $1(2,2)$ & - \\
64 & $1(2,2 \%)$ & - & - \\
192 & $1(2,2 \%)$ & - & - \\
256 & $13(28,2 \%)$ & - & \\
\hline
\end{tabular}

CIM a metronidazol $\geq 8 \mathrm{mg} / \mathrm{l}$, CIM a claritromicina y amoxicilina $\geq 2 \mathrm{mg} / \mathrm{l}$ fueron considerados resistentes. 
de la gastritis no atrófica, 33,3\% (4/12) de la úlcera duodenal, 33,3\% (4/12) de los casos de lesiones preneoplásicas y $50 \%$ (1/2) de los cáncer gástrico.

\section{DisCUSIÓN}

En el año 2000, el NCCLS estableció normas estandarizadas para la determinación de la sensibilidad a antibióticos en $\mathrm{H}$. pylori por el método de dilución en $\operatorname{agar}^{15}$, el cual es un método laborioso, indicado para el procesamiento simultáneo de gran número de cepas y no aplicable de forma rutinaria. En este estudio se utilizó el método $\mathrm{E}$ test (método epsilómetro), que es más costoso, de fácil realización y puede ser utilizado en laboratorios de rutina. Si bien existían discrepancias en los resultados obtenidos, especialmente para metronidazol ${ }^{19,20}$, estudios recientes demostraron que hay muy buena correlación entre el método epsilómetro y el de dilución del antibiótico en $\operatorname{agar}^{16}$.

En nuestro estudio, la prevalencia de cepas de $\mathrm{H}$. pylori resistentes a metronidazol fue alta $(32,6 \%)$ y baja para claritromicina y amoxicilina (2,2\%). La resistencia de $\mathrm{H}$. pylori a metronidazol es muy variable en los diferentes países y han sido reportadas resistencias con rangos que oscilan entre $5 \%$ y $90 \%{ }^{11}$. La alta prevalencia de cepas resistentes de $\mathrm{H}$. pylori al metronidazol en nuestro país es comparable a los valores obtenidos en otros países en vías de desarmollo, como Chile ${ }^{21}$. Con esta prevalencia, se debe tener presente que si se opta por un esquema terapéutico que incluya metronidazol podńa resultar en una falla del tratamiento. La vigilancia de la resistencia a metronidazol es importante, porque este medicamento es comúnmente usado en muchos esquemas terapéuticos ${ }^{22}$.

La clanitromicina tiene mejor propiedad farmacocinética que otros macrólidos, es más estable que la enitromicina en medio ácido y penetra en altas concentraciones en el tejido gástrico y mucus y tiene vida media más larga ${ }^{20}$. La resistencia a claritromicina observada en las cepas de $\mathrm{H}$. pylori aisladas en Paraguay, no difiere mayormente de lo informado en la mayoría de los países, que han reportado porcentajes de cepas resistentes a clanitromicina en menos de $10 \%{ }^{21}$. Consideramos que los valores bajos de concentración inhibitoria mínima que hemos obtenido para la clanitromicina y el bajo porcentaje de resistencia, hace que este antibiótico pueda ser utilizado en el esquema inicial para la erradicación del $\mathrm{H}$. pylori en nuestro país. La resistencia a claritromicina suele estar muy relacionada con el uso de macrólidos, de ahí la variabilidad en la resistencia según las diferentes zonas geográficas. Aunque el porcentaje de cepas resistentes a claritromicina aisladas en nuestro país es bajo, debemos mantener una vigilancia epidemiológica, ya que la resistencia a macrólidos se disemina rápidamente en la población y existe resistencia cruzada entre ellos ${ }^{23}$.

En cuanto a la amoxicilina, además de presentar CIM bajas, tiene excelente difusión en el jugo gástrico, donde alcanza altas concentraciones. En nuestro estudio obtuvimos valores de CIM bajos, con un alto porcentaje de sensibilidad, como se ha informado en numerosos trabajos ${ }^{4}$, por lo que se sugiere que este antibiótico sea utilizado en el esquema inicial del tratamiento para H. pylori, sin embargo, debe considerarse que esta bacteria puede presentar tolerancia a la droga ${ }^{24}$.

El aislamiento de $\mathrm{H}$. pylori es, sin duda, el método más específico en el diagnóstico de este microorganismo. A pesar de ser un método tedioso, de difícil realización debido a que $\mathrm{H}$. pylori es un microorganismo considerado fastidioso, es importante efectuar el cultivo, ya que además de determinar la sensibilidad a los antimicrobianos, permite la caracterización de factores de virulencia ${ }^{9}$.

Actualmente existe consenso internacional para el tratamiento de todas las infecciones producidas por H. pylori y el mayor obstáculo para el éxito terapéutico es la resistencia a los antimicrobianos. Consideramos que los resultados obtenidos en este primer estudio sobre resistencia a los antibióticos en cepas paraguayas de $\mathrm{H}$. pylori, pueden ser de gran utilidad para la selección del esquema inicial de tratamiento a ser utilizado en Paraguay. Por otro lado, un tratamiento eficaz de erradicación de $\mathrm{H}$. pylori mediante un adecuado esquema, es fundamental para evitar la progresión de las gastritis con alteraciones importantes como la gastritis atrófica multifocal con o sin metaplasia intestinal a displasia y cáncer gástrico. En caso de falla terapéutica, es imprescindible realizar cultivo y prueba de sensibilidad antes de iniciar un nuevo tratamiento.

Con el propósito de darle robustez a los datos de resistencia a antibióticos encontrados en este estudio, es necesario desarrollar un estudio a mayor escala y con muestras obtenidas de otras regiones de la nación. 
Agradecimientos

A la IARC (Agencia Internacional para la Investigación del Cáncer de la Organización Mundial de la Salud) y al equipo paraguayo del Proyecto Internacional de Helicobacter pylori, por el apoyo en la provisión de biopsias gástricas y materiales para el cultivo y aislamiento de H. pylori.

A la Dra. Dulciene Queiroz y demás miembros del Laboratorio de Investigación en Bacteriología,

\section{REFERENCIAS}

1. FeRnándeZ H. Helicobacter pylori. Un nuevo agente a ser considerado en patología gástrica. Cuad Cir 1993; 7: 91-7.

2. MarshaLl BJ, Warren JR. Unidentified curved bacilli in the stomach of patients with gastritis and peptic ulceration. Lancet 1984; i: 1311-15.

3. Dunn B, Cohen H, Blaser M. Helicobacter pylori. Clin Microbiol Rev 1997; 10(4): 720-41.

4. Kasamatsu E, Centurión M, Macchi H, Laterza O, Ortiz Vilalba J, Martínez C. Evaluación de las lesiones histológicas en las gastritis crónicas asociadas a Helicobacter pylori. Ann Rep IICS 1995: 21-37.

5. Internacional Agency for Research on Cancer, IARC monographs on the evaluation of carcinogenic risks to humans. Schistosomes, Liver Flukes and Helicobacter pylori. Lyon, IARC 1994; 61: 177-240.

6. CoRrea P. Helicobacter pylori and gastric carcinogenesis. Am J Surg Pathol 1995; 19 Suppl 1: S37-43.

7. Mera R, Fontham TH, Bravo LE, Bravo JC, Piazuelo MB, CAMARgo MC, Correa P. Long term follow up of patients treated for Helicobacter pylori infection. Gut 2006; 55(7): 1536-40.

8. Cuchí Burgos E, Forné Bardera M, Quintana Riera S, Lité Lité J, Garau Alemany J. Evolución de la sensibilidad de 235 cepas de Helicobacter pylori entre 1995 y 1998 e impacto del tratamiento antibiótico. Enferm Infecc Microbiol Clin 2002; 20(4): 157-60.

9. Alarcón T, Baquero M, Domingo D, López-Brea M, Royo G. Diagnóstico microbiológico de la infección por Helicobacter pylori. 2004. Procedimientos en Microbiología Clínica. SEIMC, Madrid 2004. de la Facultad de Medicina de la Universidad Federal de Minas Geráis, Belo Horizonte, Brasil, por el entrenamiento en el cultivo e identificación de $\mathrm{H}$. pylori y a la UICC (Unión Internacional contra el Cáncer) por el apoyo financiero para dicho entrenamiento.

Al Laboratorio Central de Salud Pública de Paraguay por la provisión de la cepa ATCC 43504 de H. pylori.

10. Glupczynski Y, BuRetTe A. Clinical Review: drug therapy for $\mathrm{H}$. pylori infection: problemas and pitfalls. Am J Gastroenterol 1990; 85: 1545-51.

11. Goddard AF, Logan RPH. Antimicrobial resistance and Helicobacter pylori. J Antimicrob Chemother 1996; 37: 639-43.

12. Loo V, FaLone C, De Souza E, Lavalée J, Barkun A. In-vitro susceptibility of Helicobacter pylori to ampicillin, clarithromycin, metronidazole and omeprazole. J Antimicrob Chemother 1997; 40: 881-3.

13. Flores S, Opazo H, Valderrama D, Aguilera $R$, Marchese A, ValderRama S. Terapia triple de corto tiempo con azitromicina, amoxicilina y omeprazol para la erradicación de Helicobacter pylori. Rev Méd Chile 2000; 128: 509-12.

14. Kalach N, Bergeret M, Benhamou PH, Dupont C, RaYMond J. High levels of resistance to metronidazole and clarithromycin in Helicobacter pylori strains in children. J Clin Microbiol 2001; 39: 394-7.

15. National Committee for Clinical Laboratory Standards. 2000. Performance standards for antimicrobial susceptibility testing and approved standard M7-A5. Informational supplement M100-S10.22. National Committee for Clinical Laboratory Standards, Wayne, Pa.

16. Best LM, Haldane DJM, Keelan M, Taylor DE, Thomson ABR, Loo V et al. Multilaboratory Comparison of Proficiencies in Susceptibility Testing of Helicobacter pylori and Correlation between Agar Dilution and E Test Methods. Antimicrob Agents Chemother 2003; 47: 3138-44.

17. Queiroz DMM, MeNDes EN, Rocha GA. Indicator medium for isolation of Campylobacter pylori. J Clin Microbiol 1987; 25: 2378-9.

18. Queiroz DM, Mendes EN, Rocha GA, Moura SB, Resende LM, Barbosa AJ et al. Effect of Helicobac- 
ter pylori eradication on antral gastrin and somatostatin-immunoreactive cell density and gastrin and somatostatin concentrations. Scand J Gastroenterol 1993; 28: 858-64.

19. Glupczynski Y, Broutet N, Cantagrel A, Andersen LP, Alarcon V, López-Brea V, Megraud F. Comparison of the $E$ test and agar dilution method for antimicrobial suceptibility testing of Helicobacter pylori. Eur J Clin Microbiol Infect Dis 2002; 21: 549-52.

20. Piccolomini R, Bonaventura G Di, Catamo G, CarboNE F, NERI M. Comparative evaluation of the E test, agar dilution, and broth microdilution for testing susceptibilities of Helicobacter pylori strains to 20 antimicrobial agents. J Clin Microbiol 1997; 35: 1842-6.

21. González C, García A, Daroch F Kawaguchi F, Solar H, Rivera N, Vega E. Susceptibilidad in vitro de cepas de Helicobacter pylori: aislamiento de cepas resistentes a claritromicina. Rev Méd Chile 2001; 129: 643-6.

22. SoL AH. Consensus conference. Medical treatment of peptic ulcer disease. Practice guidelines. JAMA 1996; 275: 622-9.

23. Vásquez Y, Valdés R, Guman JJ, Mc Donald TU, Westblom D, Berg D et al. Metronidazole and clarithromycin resistance in Helicobacter pylori. Determined by measuring MICs of Antimicrobial agents in color indication egg yolk agar in a miniwell format. J Clin Microbiol 1996; 34: 1232-4.

24. Dore MP, Sepúlveda AR, Mura I, Realdi G, Osato MS, Graham DY. Explanation for variability of omeprazole amoxicillin therapy? Tolerance of Helicobacter pylori to amoxicillin. Gastroenterol 1997; 112: A105. 